\title{
Two-level Indoor Navigation using WiFi and MEMS Sensors
}

\author{
Yuliang Huang, Yongbo Zhang*, Yi Cui, Zhihua Wang and Huimin Fu \\ Research Center of Small Sample Technology, Beihang University, Beijing 100191, China \\ ${ }^{*}$ Corresponding author
}

\begin{abstract}
In this paper, the indoor pedestrian location algorithm is investigated, in which we proposes a novel WiFi/MEMS integration structure for indoor navigation to fuse the information from WiFi and MEMS sensors. In WiFi part, aiming at the problem of large amount of calculation in the algorithm, a partition solution called "moving partition" is originally proposed. The test results from indoor experiments indicate that with "moving partition", the computation time decreased by almost half and the proposed WiFi/MEMS solution works well, of which the mean positioning accuracy has been greatly improved compared with MEMS alone and WiFi alone.
\end{abstract}

Keywords-indoor navigation, MEMS sensors, WiFi, extended kalman filter

\section{INTRODUCTION}

In recent years, location-based services (LBS) have become increasingly important. They are required in various fields such as mobile commerce, parcel or vehicle tracking, discovering the nearest shops or restaurants, and social networking [1]. The demand for indoor navigation is increasing rapidly in a number of applications. Therefore, indoor navigation has gained plenty of attention from companies and researchers.

While Global Navigation Satellite Systems (GNSS) based outdoor positioning and navigation has achieved great advances in the past few decades, indoor navigation still remains an unsolved problem [2]. The main challenges consist of the unavailability or degradation of GNSS signals, the complexity of indoor environments, and the necessity of using low-grade devices [3]. Under such a circumstance, various positioning techniques have been researched, such as IEEE 802.11 WLAN (WiFi) [4], Radio Frequency Identification (RFID) [5], ultrasound [6], Ultra Wideband Beacons (UWB) [7], Bluetooth [8], ZigBee [9], infrared [10], pseudolites [11] and pedestrian dead-reckoning (PDR) based inertial sensors.

Different positioning techniques have different advantages and disadvantages. For example, positioning results for the method based WiFi, BLE or ZigBee are independent of each other with low accuracy; PDR method has high accuracy in a short time but with serious error accumulation. So integrated navigation is a good way to combine various technologies to achieve the purpose of high accuracy and low cost at the same time. For the integration of WiFi and MEMS solutions, the literature [12] uses the robust extended Kalman filter (EKF) to fuse the information from WiFi/MEMS sensors in a multi-floor environment, while the research [13] presents a pedestrian navigator based on tightly coupled integration of low-cost MEMS sensors and WiFi for handheld devices. Using the same data from WiFi/MEMS sensors, various information fusion structures may lead to different results [3]. This paper proposes a novel partitioning algorithm to reduce the computing time of the system and a novel two-level structure to fuse the information from WiFi/MEMS sensors.

\section{WIFI-BASED NAVIGATION}

The whole process of WiFi fingerprinting is depicted in Fig. 1. In fingerprinting scheme, to estimate the position of the MU, various matching algorithms have been proposed [14]. For example, neural network algorithm, support vector machine algorithm and Weighted K-Nearest Neighbors (WKNN) method.

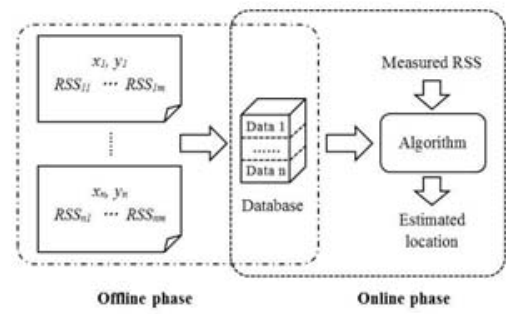

FIGURE I. WIFI FINGERPRINTING APPROACH

Weighted K-Nearest Neighbors (WKNN) method [15] is a simple, performing well and commonly used method. However, it needs to search the overall space and go through the whole database fingerprints. For a big place and a huge database, in order to obtain higher computational efficiency, in the current study, a partition solution is proposed and called "moving partition". As shown in Fig. 2, a circle located at the previous point is drawn with the given radius, and a partition in the circle is formed. Suppose there are 10 RPs in the circle. These RPs are stored in an array. The current point is calculated within the scope of the 10 RPs using the WKNN method. Once the current point is located, a new partition is got with the same radius as the old one. The method to build the new partition is removing the last RPs in the array and putting in the new ones. The number of the changing RPs is determined by Eq. (4).

$$
n=\left[\frac{m|R-r|}{L}\right]
$$

where $[x]$ means the maximum integer that is no larger than $x ; L$ is the distance between adjacent RPs; $m$ is the number of RPs in a row; $R$ is the radius of the partition circle; $r$ is the 
distance between the current point and the RP at the edge of the circle in the forward direction. If $R \geq r$, the partition moves forward. If $R<r$, it moves backward. Then the next point is calculated within the scope of RPs in the new partition.

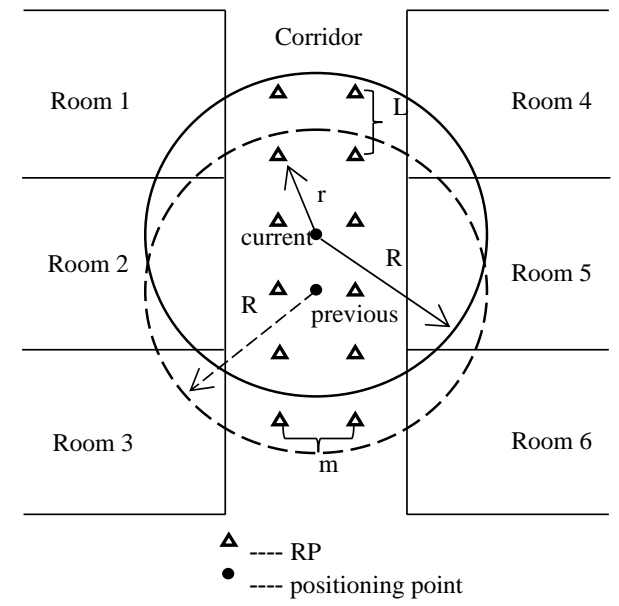

FIGURE II. MOVING PARTITION.

With "moving partition”, the area of search space is greatly reduced. In addition, the work to update the partition is greatly reduced as well. Therefore, the computation time decreases significantly. In the current study, the size of test area is around $30 \times 20 \mathrm{~m}^{2}$ and the loop corridor is $1.8 \mathrm{~m}$ wide. There were 158 RPs and 155 positioning points in all. Test results are illustrated in Table I. As shown, the running time of "moving partition" and the traditional WKNN method on the same device was $0.33 \mathrm{~s}$ and $0.64 \mathrm{~s}$, respectively. With "moving partition", the computation time decreased by almost half.

TABLE I. TEST RESULTS

\begin{tabular}{ccc}
\hline & moving partition & WKNN \\
\hline running time, $\mathrm{s}$ & 0.33 & 0.64 \\
average positioning error, $\mathrm{m}$ & 1.92 & 1.98 \\
\hline
\end{tabular}

\section{SENSOR-BASED NAVIGATION}

\section{A. Step Detection}

Methods of step detection include peak detection, zero crossings, auto-correlation or template matching, and spectral analysis [16]. In the current study, peak detection is utilized.

\section{B. Step Length Estimation}

Step length estimation is used to estimate the moving distance of the pedestrian at each step. In the current study, the practical model presented in [17] is utilized to estimate step length. This model assumes that the step length is proportional to the vertical movement of the human hip. The largest difference of the vertical acceleration at each step is used to calculate vertical movement [13]. The step length is estimated as follows. where $a_{z \max }$ and $a_{z \min }$ are the maximum and minimum values of the vertical acceleration, respectively; $K$ is a calibrated constant parameter. In the current study, the value of $K$ is obtained through experimental training.

\section{EKF-BASED INTEGRATION}

\section{A. Direction From WiFi and Turn Detection}

In the current study, the direction measurement of MU is estimated using the information of 4 latest positioning points from WiFi. If the MU walks straight, this method works. However, it becomes invalid at corner. Therefore, a "turn detection" is necessary before the direction estimation. We can use gyroscope data to detect whether or not to turn. It is important to note that the mobile phone must be kept as horizontal as possible during the detection [18].

\section{B. Extended Kalman Filter}

The EKF is usually utilized to fuse other information to reduce the drift of MEMS-based navigation approach. In this paper, a novel two-level EKF structure to fuse the information from WiFi/MEMS sensors is proposed.

In the first level, the information from the gyroscope and the accelerometer is fused to calculate the Euler angles.

In the second level, the information from PDR and WiFi is fused to calculate the position.

\section{EXPERIMENTS DESCRIPTION AND RESULTS}

To evaluate the performance of the proposed indoor navigation method, several experiments were performed in the ninth floor of Block C, New Main Building at Beihang University, as shown in Fig. 3.

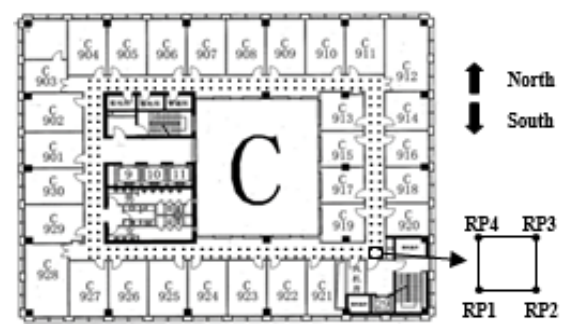

FIGURE III. FLOOR LAYOUT

Fig. 4 depicts the navigation performance comparison of WiFi, MEMS, and WiFi/MEMS integration. The "Ref" represents the actual trajectory which the tester walked along. As shown, the estimation of WiFi/MEMS integration has a good performance, making a good fit with the reference trajectory. Also, compared with $\mathrm{WiFi}$, the phenomenon of reciprocating motion has been eliminated significantly. This figure depicts the estimation result of MEMS as well. As is known, the estimation error accumulates quickly for low grade MEMS sensors in smart devices which usually have large accelerometer and gyroscope biases.

$$
S=\sqrt[4]{a_{z \max }-a_{z \min }} \cdot K
$$




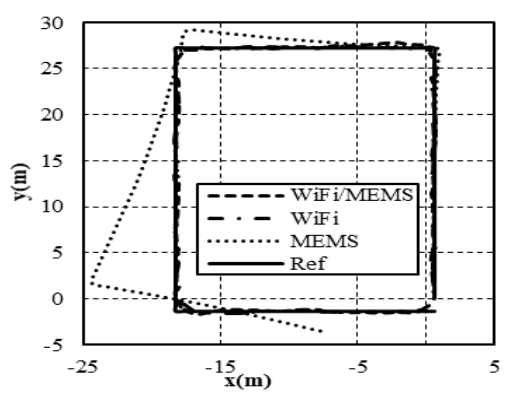

FIGURE IV. POSITIONING RESULTS.

The detailed estimation errors of these algorithms are listed in Table II. As shown, the maximum error of WiFi/MEMS is $5.05 \mathrm{~m}$, which is $64.9 \%$ of MEMS and $96.7 \%$ of $\mathrm{WiFi}$. The mean error of WiFi/MEMS is $1.71 \mathrm{~m}$, which is $61.8 \%$ of MEMS and $89.0 \%$ of WiFi. Therefore, the EKF-based WiFi/MEMS algorithm proposed in this paper has improved the estimation accuracy obviously.

TABLE II. POSITIONING ERRORS

\begin{tabular}{cccc}
\hline & Minimum, $\mathrm{m}$ & Maximum, $\mathrm{m}$ & Mean, $\mathrm{m}$ \\
\hline WiFi & 0.03 & 5.22 & 1.93 \\
MEMS & 0.04 & 7.78 & 2.77 \\
WiFi/MEMS & 0.06 & 5.05 & 1.71 \\
\hline
\end{tabular}

Furthermore, the error probabilities of these algorithms are depicted in Fig. 5. As shown, the cumulative error percentages of $\mathrm{WiFi}$ and $\mathrm{WiFi} / \mathrm{MEMS}$ are close when the error is less than $1.4 \mathrm{~m}$, but WiFi/MEMS has quickly approached the best performance when the error is larger than the mean error, showing a very stable estimation.

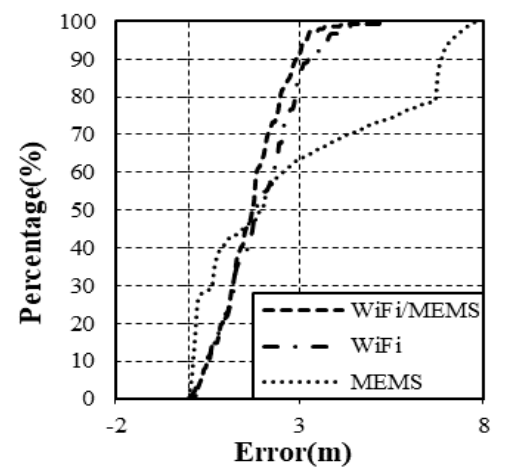

FIGURE V. CUMULATIVE ERROR PERCENTAGES

\section{CONCLUSION}

This paper presents a novel WiFi/MEMS fusion structure for indoor navigation. In WiFi fingerprinting scheme, a partition solution called "moving partition" is originally proposed in this paper. With "moving partition", the computation time decreases by almost half compared with the traditional WKNN method. Also, the direction measurement of MU using the information of 4 latest positioning points from WiFi is presented, and a "turn detection" is applied before the direction fusion. Then a novel two-level structure to integrate the information from WiFi/MEMS sensors using the EKF is proposed.
With these improvements, the navigation performance of the proposed WiFi/MEMS integration solution is compared with results from WiFi and MEMS. Experiment results show that the mean position error of the proposed WiFi/MEMS solution is $1.71 \mathrm{~m}$, which is $61.84 \%$ of MEMS and $88.99 \%$ of WiFi.

\section{REFERENCES}

[1] B. Shin, J. H. Lee, T. Lee, and H. S. Kim, "Enhanced weighted K-nearest neighbor algorithm for indoor Wi-Fi positioning systems," Int J. Netw. Comput. Adv. Inf. Manage., vol. 2, no. 2, pp. 15-21, Oct. 2012.

[2] X. Li, J. Wang, and T. Li, "Seamless positioning and navigation by using geo-referenced images and multi-sensor data," Sens., vol. 13, no. 7, pp. 9047-9069, Jul. 2013.

[3] Y. Li, Y. Zhuang, P. Zhang, H. Lan, X. Niu, and N. El-Sheimy, "An improved inertial/wifi/magnetic fusion structure for indoor navigation," Inf. Fusion, vol. 34, pp. 101-119, Jul. 2016.

[4] G. Caso and L. D. Nardis, "Virtual and oriented WiFi fingerprinting indoor positioning based on multi-wall multi-floor propagation models," Mobile Netw. Appl., vol. 22, pp. 825-833, 2017.

[5] S. Seol, E. K. Lee, and W. Kim, "Indoor mobile object tracking using RFID,” Future Gener. Comput. Syst., vol. 76, pp. 443-451, 2017.

[6] A. Lindo, E. García, J. Ureña, M. C. Pérez, and A. Hernández, "Multiband waveform design for an ultrasonic indoor positioning system,” IEEE Sens. J., vol. 15, no. 12, pp. 7190-7199, Dec. 2015.

[7] P. Chen, Y. Kuang, and X. Chen, “A UWB/improved PDR integration algorithm applied to dynamic indoor positioning for pedestrians,” Sens., vol. 17, no.9, Sep. 2017.

[8] C. Zhou, J. Yuan, H. Liu, and J. Qiu, "Bluetooth indoor positioning based on RSSI and Kalman filter,” Wireless Pers. Commun., vol. 96, pp. 4115-4130, Jul. 2017.

[9] M. Aykaç, E. Erçelebi, and N. B. Aldin, “ZigBee-based indoor localization system with the personal dynamic positioning method and modified particle filter estimation,” Analog Integr. Circ. Sig. Process., vol. 92, pp. 263-279, Apr. 2017.

[10] K. Wang, A. Nirmalathas, C. Lim, K. Alameh, H. Li, and E. Skafidas, "Indoor infrared optical wireless localization system with background light power estimation capability,” Opt. Express, vol. 25, no. 19, Sep. 2017.

[11] X. Li, P. Zhang, J. Guo, J. Wang, and W. Qiu, "A new method for single-epoch ambiguity resolution with indoor pseudolite positioning," Sens., vol. 17, no. 4, Apr. 2017.

[12] Y. Zhuang and N. El-Sheimy, "Tightly-coupled integration of WiFi and MEMS sensors on handheld devices for indoor pedestrian navigation,” IEEE Sens. J., vol. 16, no. 1, pp. 224-234, Jan. 2016.

[13] Z. Tian, X. Fang, M. Zhou, and L. Li, "Smartphone-based indoor integrated WiFi/MEMS positioning algorithm in a multi-floor environment,” Micromach., vol. 6, pp. 347-363, Mar. 2015.

[14] S. Khodayari, M. Maleki, and E. Hamedi, "A RSS-based fingerprinting method for positioning based on historical data," in Proc. Int. Symp. Perform. Eval. Comput. Telecommun. Syst., Ottawa, ON, Canada, 2010, pp. 306-310.

[15] L. M. Ni, Y. Liu, Y. C. Lau, and A. P. Patil, "LANDMARC: Indoor location sensing using active RFID,” in Proc. 1st IEEE Int. Conf. Pervasive Comput. Commun., Fort Worth, TX, USA, 2003.

[16] R. Harle, "A survey of indoor inertial positioning systems for pedestrians,” IEEE Commun. Surv. Tutor., vol. 15, no. 3, pp. 1281-1293, 2013.

[17] H. Weinberg, "Using the ADXL202 in pedometer and personal navigation applications," Analog Devices, Norwood, MA, USA, Appl. Note AN-602, 2002.

[18] C. Liu, "Study on the pedestrian indoor positioning algorithms based on WIFI and inertial technology,” M.S. thesis, China Univ. Min. Technol., Xuzhou, China, 2015. 